\section{A new method to facilitate bilateral hepatic duct drainage: simultaneous insertion of two thin $6-\mathrm{Fr}$ self-expandable metal stents}

An uncovered biliary self-expandable metal stent (SEMS) mounted on a 6-Fr delivery catheter has become available. It allows simultaneous side-by-side insertion of two SEMSs through the working channel of a standard therapeutic duodenoscope.

A patient with a hilar stricture related to a gallbladder carcinoma was referred for biliary drainage. A standard duodenoscope (TJF-160; Olympus, Tokyo, Japan) was used for the whole procedure ( $\mathbf{F i g} \mathbf{1}$ ).

Bilateral duct opacification was performed, followed by balloon dilation (6-mm Hurricane balloon catheter; Boston Scientific, Natick, Massachusetts, USA) of the left and right hepatic ducts, and failed insertion of two Wallflex SEMSs (Boston
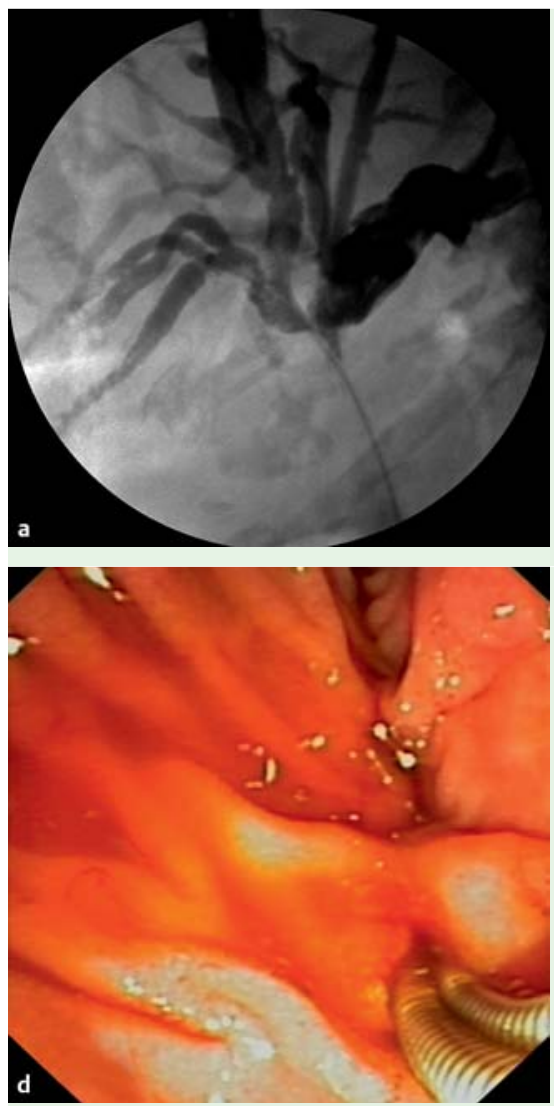

Scientific), which were removed. Immediately after SEMS removal, during the same endoscopic retrograde cholangiopancreatography (ERCP), two Zilver 635 SEMSs (Cook Medical, Winston-Salem, North Carolina, USA) were inserted, using the two guide wires that had been used for Wallflex insertion and without repeated stricture dilation. The Zilver SEMSs were deployed very slowly (the Zilver 635 is a nonshortening SEMS that deploys less gradually than the Wallflex, and its recapture into the delivery catheter is not possible). Serum bilirubin levels returned to normal values. No post-procedure complications were detected.

The procedure was successfully repeated in another patient with a Bismuth IV hilar
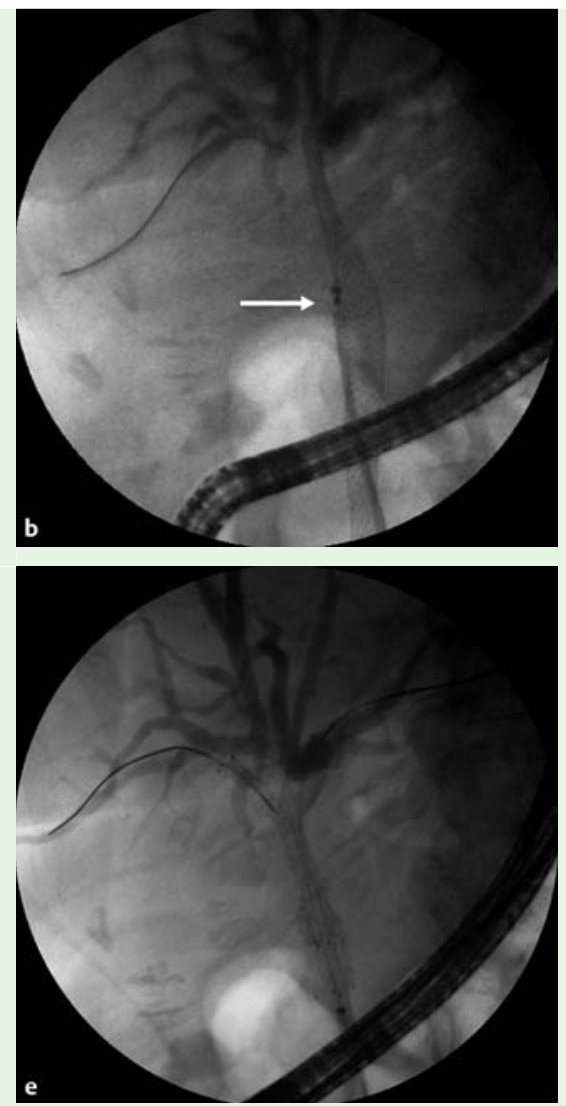

stricture related to hepatocellular carcinoma (two Zilver 635 SEMSs were deployed simultaneously without any prior attempt with other SEMSs).

Only the Zilver 635 model is suitable for this technique; the standard Zilver model supplies an identical SEMS mounted on a 7-Fr transparent delivery catheter, allowing endoscopic control of transpapillary SEMS release.

The technique described may be particularly useful if two major ducts have been injected with contrast medium, to avoid leaving contaminated ducts undrained [1], and could potentially modify our approach to endoscopic drainage of hilar strictures. Simultaneous side-by-side insertion of two standard SEMSs into the hilum has been reported, but it requires a duodenoscope that is not yet commercially available, with a 6-mm diameter working channel [2].

\section{Endoscopy_UCTN_Code_TTT_1AR_2AZ}

\section{Competing interests: None}
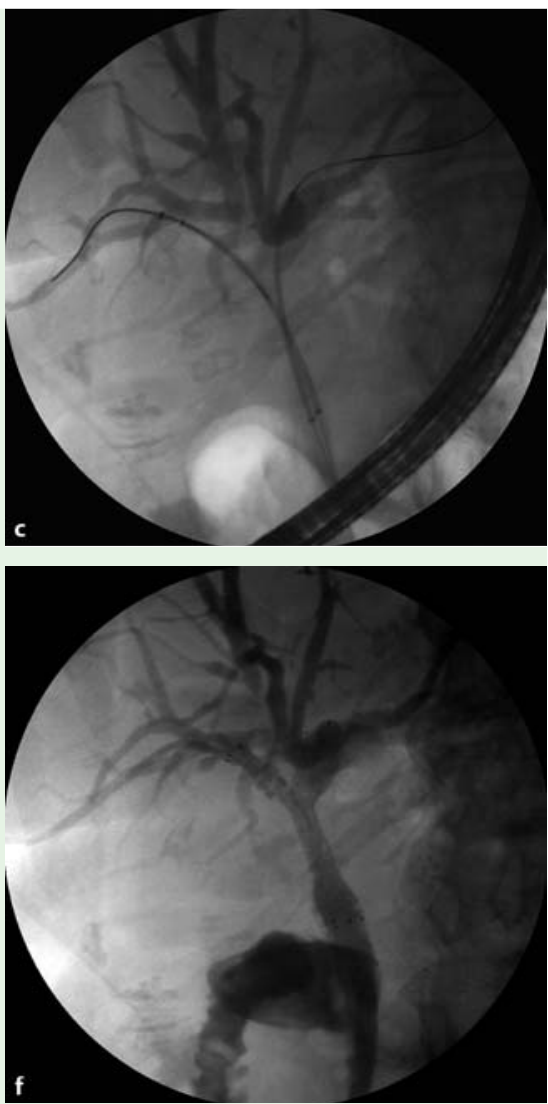

Fig. 1 a Cholangiogram showing a Bismuth III hilar stricture. b A $10 \times 100-m m$ uncovered Wallflex self-expandable metal stent (SEMS; Boston Scientific) is partially deployed in the left hepatic duct; the radiopaque marker (arrow) of the second, constrained, identical Wallflex SEMS indicates the most proximal point that could be reached while attempting SEMS insertion into the right hepatic duct. c Immediately after endoscopic removal of the two Wallflex SEMSs (using a snare for the released SEMS), the two guide wires that had previously been used were reinserted bilaterally, and two Zilver 635 SEMSs ( $10 \times 60$ mm; Cook Medical) were advanced sequentially, side by side, and positioned across the strictures. $\mathbf{d}$ Endoscopic view of the two delivery catheters inserted simultaneously into the papilla. e The two Zilver SEMSs in place after simultaneous deployment by two assistants. (Deployment was extremely progressive, alternating the SEMSs for one third of the length of each up to final release.) f Contrast medium draining through the SEMSs into the duodenum. 


\section{T. Nguyen-Tang, J.-M. Dumonceau}

Division of Gastroenterology and Hepatology, Geneva University Hospitals, Geneva, Switzerland

\section{References}

1 Sherman S. Endoscopic drainage of malignant hilar obstruction: is one biliary stent enough or should we work to place two? Gastrointest Endosc 2001; 53: 681 - 684

2 Saleem A, Baron TH. Large-diameter therapeutic channel duodenoscope to facilitate simultaneous deployment of side-by-side self-expandable metal stents in hilar cholangiocarcinoma. Gastrointest Endosc 2010; 72: $628-631$

\section{Bibliography}

DOI $10.1055 / \mathrm{s}-0030-1255945$

Endoscopy 2011; 43: E24-E25

(c) Georg Thieme Verlag KG Stuttgart · New York . ISSN 0013-726X

\section{Corresponding author}

T. Nguyen-Tang, MD

Division of Gastroenterology and Hepatology

Geneva University Hospitals

Rue Gabrielle-Perret-Gentil 4

$\mathrm{CH}-1211$ Geneva 4

Switzerland

Fax: +41-22-3729366

thai.nguyen-tang@hcuge.ch 